\title{
Fixed point theorems in vector metric spaces for multivalued operators
}

\author{
Ioan-Radu Petre
}




\title{
FIXED POINT THEOREMS IN VECTOR METRIC SPACES FOR MULTIVALUED OPERATORS
}

\author{
IOAN-RADU PETRE
}

Received 06 April, 2014

\begin{abstract}
In this work we prove some extensions of the multivalued contraction principle and of Reich's fixed point theorem for multivalued operators in vector metric spaces. We also present a theory for the multivalued contraction principle in vector metric spaces in the spirit of the approach given by I. A. Rus and A. Petruşel. Some examples and open questions are pointed out.

2010 Mathematics Subject Classification: 47H10; 54H25

Keywords: multivalued contraction principle, multivalued Reich contraction, contractive mapping, data dependence, fixed point, strict fixed point, generalized contraction, Hausdorff convergence, limit shadowing property, Picard operator, Riesz space, multivalued operator, vector metric space, weakly Picard operator, well-posedness of fixed point problem
\end{abstract}

\section{Preliminaries}

We present first some auxiliary concepts and results, see the monograph of Aliprantis and Border [1] and the manuscripts $[4,7,18]$.

A partially ordered set $(X, \geq)$ is a lattice if each pair of elements $x, y \in X$ has a supremum and an infimum.

A real vector space $E$ with an order relation $\geq$ on $E$ that is compatible with the algebraic structure of $E$ in the sense that satisfies properties:

$$
\begin{aligned}
& \text { (1) } x \geq y \text { implies } x+z \geq y+z \text { for each } z \in E, x, y \in E \text { and } \\
& \text { (2) } x \geq y \text { implies } t x \geq t y \text { for each } t>0, x, y \in E
\end{aligned}
$$

is called an ordered vector space.

An ordered vector space that is also a lattice is called a Riesz space or vector lattice. Many familiar spaces are Riesz spaces, as is shown by the following standard examples (see $[1,4])$ :

(1) The space $\mathbb{R}^{n}$ with the Euclidean norm and with the componentwise ordering relation is a Riesz space.

(2) Given a compact Hausdorff space $T$, the vector space $C(T)$ of all continuous real functions and the vector space $C_{b}(T)$ of all bounded continuous real functions with the supremum norm and with the pointwise ordering relation 
are Riesz spaces. In particular, the space $c$ of convergent real sequences and the space $c_{0}$ of real null-sequences are Riesz spaces.

(3) Given a measure space $(X, A, \mu)$ and $1 \leq p \leq \infty$, the Lebesgue space $L_{p}(\mu)$ is a Riesz space with the standard $\|\cdot\|_{p}$ norm and the almost everywhere pointwise ordering relation. In particular, the $\ell_{p}$ spaces of real sequences are also Riesz spaces.

The symbolistic $x_{n} \downarrow x$ means that $x_{n}$ is a decreasing sequence and $\inf \left\{x_{n}\right\}=x$. Some basic properties of decreasing sequences are:

. $x_{n} \downarrow x$ and $y_{m} \downarrow y$ implies $x_{n}+y_{m} \downarrow x+y$;

- $x_{n} \downarrow x$ implies $\lambda x_{n} \downarrow \lambda x$, for $\lambda>0$ and $\lambda x_{n} \uparrow \lambda x$, for $\lambda<0$;

- $x_{n} \downarrow x$ and $y_{m} \downarrow y$ implies $x_{n} \vee y_{m} \downarrow x \vee y$ and $x_{n} \wedge y_{m} \downarrow x \wedge y$.

The meaning of $x_{n} \uparrow x$ and some basic properties of increasing sequences are similar.

A Riesz space $E$ is Archimedean if $\frac{1}{n} x \downarrow 0$ holds for every $x \in E^{+}$, where

$$
E^{+}=\{x \in E: x \geq 0\} \text { is the positive cone of } E .
$$

A Riesz space $E$ is order complete or Dedekind complete if every non-empty subset of $E$ which is bounded above has a supremum. Any order complete Riesz space is Archimedean. The converse is false.

Let $E$ be a Riesz space. A sequence $\left(b_{n}\right)$ in $E$ is called order-convergent (or $o$ convergent) to $b$, if there exists a sequence $\left(a_{n}\right)$ in $E$ satisfying $a_{n} \downarrow 0$ and $\left|b_{n}-b\right| \leq$ $a_{n}$ holds for all $n$, written $b_{n} \stackrel{o}{\longrightarrow} b$, where

$$
|x|=x \vee(-x) \text { for any } x \in E .
$$

A sequence $\left(b_{n}\right)$ in $E$ is called order-Cauchy (or o-Cauchy), if there exists a sequence $\left(a_{n}\right)$ in $E$ such that $a_{n} \downarrow 0$ and $\left|b_{n}-b_{n+p}\right| \leq a_{n}$ holds for all $n$ and $p$. A Riesz space $E$ is called $o$-Cauchy complete if every $o$-Cauchy sequence is $o$-convergent.

Let $E, F$ be two Riesz spaces and $f: E \rightarrow F$. The function $f$ is order continuous (or o-continuous) if $b_{n} \stackrel{o}{\longrightarrow} b$ in $E$ implies $f\left(b_{n}\right) \stackrel{o}{\longrightarrow} f(b)$ in $F$.

For notations and other facts regarding lattice and order properties, order convergence and order continuity in Riesz spaces we refer to [1].

We present now the concept of vector metric space.

Definition 1. Let $X$ be a non-empty set and $E$ be a Riesz space. The function $d: X \times X \rightarrow E$ is said to be a vector metric (or E-metric) if it satisfies the following properties:

(a) $d(x, y)=0$ if and only if $x=y$,

(b) $d(x, y) \leq d(x, z)+d(y, z)$, for all $x, y, z \in X$.

Also, the triple $(X, d, E)$ is said to be a vector metric space. 
Example 1. A Riesz space $E$ is a vector metric space with $d: E \times E \rightarrow E$ defined by

$$
d(x, y)=|x-y| .
$$

This vector metric is called the absolute valued metric on $E$.

Now we give some useful definitions for our main results.

Let $(X, d, E)$ be a vector metric space. A sequence $\left(x_{n}\right)$ in $X$ is called vectorially converging (or E-converges) to some $x \in E$, written $x_{n} \stackrel{d, E}{\longrightarrow} x$, if there is a sequence $\left(a_{n}\right)$ in $E$ such that $a_{n} \downarrow 0$ and $d\left(x_{n}, x\right) \leq a_{n}$ for all $n$. A sequence $\left(x_{n}\right)$ in $X$ is called vectorially Cauchy (or $E$-Cauchy), if there is a sequence $\left(a_{n}\right)$ in $E$ such that $a_{n} \downarrow 0$ and $d\left(x_{n}, x_{n+p}\right) \leq a_{n}$ holds for all $n$ and $p$. A vector metric space $X$ is called $E$-complete if each $E$-Cauchy sequence in $X E$-converges to a limit in $X$. If $E=\mathbb{R}$, the concepts of $E$-convergence and metric convergence are the same, respectively the concepts of $E$-Cauchy sequence and Cauchy sequence are the same. If $X=E$ and $d$ is the absolute valued vector metric on $X$, then the concepts of vectorially convergence and convergence in order are the same. Notice than, in particular, if $X=E$ and $d$ is an absolute valued vector metric on $E$, then we obtain fixed point theorems of Riesz space $E$.

The subset $\varnothing \neq A \subseteq X$ defined by

$$
\delta(A)=\sup \{d(x, y): x, y \in A\}
$$

is called the $E$-diameter of $X$ if $\sup \{d(x, y): x, y \in A\}$ in $E$. Furthermore, if there exists an $a>0$ in $E$ such that $d(x, y) \leq a$, for $x, y \in A$, then $A$ is called an $E$ bounded set.

Let $X, Y$ be two vector metric spaces and $f: X \rightarrow Y$. The function $f$ is vectorially continuous (or E-continuous) if $x_{n} \stackrel{d, E}{\longrightarrow} x$ in $X$ implies $f\left(x_{n}\right) \stackrel{d, E}{\longrightarrow} f(x)$ in $Y$.

If $X$ is a nonempty set and $T: X \rightarrow P(X)$ is a multivalued operator, we denote by $F_{T}:=\{x \in X \mid x \in T(x)\}$, where

$$
\begin{aligned}
\mathcal{P}(X) & :=\{Y \mid Y \subseteq X\} ; \\
P(X) & :=\{Y \in \mathcal{P}(X) \mid Y \neq \varnothing\}
\end{aligned}
$$

and in the context of a vector metric space $(X, d, E)$, we denote by

$$
\begin{aligned}
P_{c l}(X) & :=\{Y \in P(X) \mid Y \text { is } E \text {-closed }\} ; \\
P_{b}(X) & :=\{Y \in P(X) \mid Y \text { is } E \text {-bounded }\} ; \\
\operatorname{Graph}(T) & :=\{(x, y) \in X \mid y \in T(x)\} .
\end{aligned}
$$




\section{FIXED POINT THEOREMS FOR CONTRACTIONS AND GENERALIZED CONTRACTIONS IN VECTOR METRIC SPACES}

In this paragraph, we prove the Contraction Principle and Reich fixed point theorem for multivalued operators in vector metric spaces. Also, examples are given to obtain the existence of the fixed point using the new results.

Remark that there exists on the market some powerful background for our study, the papers of Berinde [2], Daffer \& Kaneko [5], Kaneko [8,9], Mizoguchi \& Takahashi [10], and more others obtain in their results same conclusions, but not in a vector metric space under an Archimedean assumption. Notice that in the setting of a vector metric space can appear some difficulties, especially when we want to give an example or an application of our result to a differential inclusion. For example, in the case when the vector metric space is $\mathbb{R}^{n}$, we need to introduce an absolute value property for a matrix which converges to zero to can give an application to a differential inclusions system, see I.-R. Petre [12] (Theorem 2.2), in the case when the vector metric space is a Riesz space $E$, for a working application we need to assume that $E$ is order complete which guarantees that a certain infimum exists in $E$, see I.-R. Petre [11] (Theorem 2.28), and also for an application in generalized b-metric spaces, see I.-R. Petre, M. Bota [13] (Theorem 3.13).

Definition 2. Let $(X, d, E)$ be a vector metric space. The operator $T: X \rightarrow$ $P_{c l}(X)$ is said to be a multivalued $k$-contraction, if and only if $k \in[0,1)$ and for any $x, y \in X$ and any $u \in T(x)$, there exists $v \in T(y)$ such that

$$
d(u, v) \leq k d(x, y) .
$$

Definition 3. Let $(X, d, E)$ be a vector metric space and $T: X \rightarrow P(X)$ be a multivalued operator. The sequence $\left(x_{n}\right)_{n \in \mathbb{N}} \subset X$, recursively defined by

$$
\left\{\begin{array}{l}
x_{0}=x, x_{1}=y ; \\
x_{n+1} \in T\left(x_{n}\right), \text { for all } n \in \mathbb{N},
\end{array}\right.
$$

is called the sequence of successive approximations of $T$ starting from $(x, y) \in$ $\operatorname{Graph}(T)$.

Theorem 1. Let $(X, d, E)$ be an E-complete vector metric space with $E$ Archimedean and let $T: X \rightarrow P_{c l}(X)$ be a multivalued $k$-contraction. Then $T$ has a fixed point in $X$ and for any $x \in X$, there exists a sequence of successive approximations of $T$ starting from $(x, y) \in \operatorname{Graph}(T)$ which $E$-converges in $(X, d, E)$ to the fixed point of $T$.

Proof. Let $x_{0} \in X$ such that $x_{1} \in T x_{0}$. Then there exists $x_{2} \in T x_{1}$ such that $d\left(x_{1}, x_{2}\right) \leq k d\left(x_{0}, x_{1}\right)$. Thus, define the sequence $\left(x_{n}\right) \in X$ by $x_{n+1} \in T x_{n}$ and $d\left(x_{n}, x_{n+1}\right) \leq k d\left(x_{n-1}, x_{n}\right)$, for any $n \in \mathbb{N}^{*}$. Inductively, we obtain

$$
d\left(x_{n}, x_{n+1}\right) \leq k d\left(x_{n-1}, x_{n}\right) \leq \ldots \leq k^{n} d\left(x_{0}, x_{1}\right) \text {, for any } n \in \mathbb{N}^{*} .
$$


We have

$$
\begin{aligned}
d\left(x_{n}, x_{n+p}\right) & \leq d\left(x_{n}, x_{n+1}\right)+d\left(x_{n+1}, x_{n+2}\right)+\ldots+d\left(x_{n+p-1}, x_{n+p}\right) \\
& \leq\left(k^{n}+k^{n+1}+\ldots+k^{n+p-1}\right) d\left(x_{0}, x_{1}\right)=k^{n} \frac{1-k^{p}}{1-k} d\left(x_{0}, x_{1}\right) \\
& \leq \frac{k^{n}}{1-k} d\left(x_{0}, x_{1}\right)=a_{n} \cdot a:=b_{n}, \text { for any } n \in \mathbb{N}^{*} \text { and } p \in \mathbb{N}^{*},
\end{aligned}
$$

where $a_{n}=\frac{k^{n}}{1-k} \downarrow 0$ and $a=d\left(x_{0}, x_{1}\right) \in E^{+}$.

On the other hand, by $E$ Archimedean hypothesis, we have $b_{n} \downarrow 0$. So, the sequence $\left(x_{n}\right)$ is $E$-Cauchy în $X$. By the $E$-completness of $X$, there is $z \in X$ such that $x_{n} \stackrel{d, E}{\longrightarrow}$ $z$. Hence, there exists $\left(a_{n}\right)$ in $E$ such that $a_{n} \downarrow 0$ and $d\left(x_{n}, z\right) \leq a_{n}$.

We know $x_{n+1} \in T x_{n}$ for any $n \in \mathbb{N}$ and by the multivalued $k$-contraction condition it follows that there exists $u \in T z$ such that

$$
d\left(x_{n+1}, u\right) \leq k d\left(x_{n}, z\right), \text { for any } n \in \mathbb{N} .
$$

Then the following estimation holds:

$$
\begin{aligned}
d(z, u) & \leq d\left(x_{n+1}, u\right)+d\left(x_{n+1}, z\right) \\
& \leq k d\left(x_{n}, z\right)+a_{n+1} \leq(k+1) a_{n} \downarrow 0 .
\end{aligned}
$$

Thus, we have there exists $z=u \in T z$, i.e., $T$ has a fixed point in $X$.

We can give an example to show that Theorem 1 generalize Nadler's classical metric fixed point theorem.

Example 2. Let $E=\mathbb{R}^{2}$ with componentwise ordering and let

$$
X=\left\{(x, 0) \in \mathbb{R}^{2}: 0 \leq x \leq 1\right\} \cup\left\{(0, x) \in \mathbb{R}^{2}: 0 \leq x \leq 1\right\} .
$$

The mapping $d: X \times X \rightarrow E$ is defined by

$$
\begin{aligned}
& d((x, 0),(y, 0))=\left(\frac{4}{3}|x-y|,|x-y|\right), \\
& d((0, x),(0, y))=\left(|x-y|, \frac{2}{3}|x-y|\right), \\
& d((x, 0),(0, y))=\left(\frac{4}{3} x+y, x+\frac{2}{3} y\right) .
\end{aligned}
$$

Then $X$ is an $E$-complete vector metric space. Let $T: X \rightarrow P_{c l}(X)$ with $T\left(x_{1}, x_{2}\right)=$ $\left\{u\left(x_{1}, x_{2}\right), v\left(x_{1}, x_{2}\right)\right\}$, where $u, v: X \rightarrow X$ are defined by

$$
\begin{aligned}
& u((x, 0))=(0, x) \text { and } u((0, x))=\left(\frac{x}{2}, 0\right) ; \\
& v((x, 0))=(0, x) \text { and } v((0, x))=\left(\frac{x}{3}, 0\right) .
\end{aligned}
$$

We have the following possibilities 
Case 1: for any $(x, 0),(y, 0) \in X$ and any $(0, x) \in T(x, 0)$, there exists $(0, y) \in$ $T(y, 0)$

Case 2: for any $(x, 0),(0, y) \in X$ and any $(0, x) \in T(x, 0)$, there exists $\left(\frac{y}{2}, 0\right) \in$ $T(0, y)$ or $\left(\frac{y}{3}, 0\right) \in T(0, y)$

Case 3: for any $(0, x),(y, 0) \in X$ and any $\left(\frac{x}{2}, 0\right) \in T(0, x)$ or any $\left(\frac{x}{3}, 0\right) \in T(0, x)$, there exists $(0, y) \in T(y, 0)$

Case 4: for any $(0, x),(0, y) \in X$ and any $\left(\frac{x}{2}, 0\right) \in T(0, x)$, there exists $\left(\frac{y}{2}, 0\right) \in$ $T(0, y)$, respectively for any $(0, x),(0, y) \in X$ and any $\left(\frac{x}{3}, 0\right) \in T(0, x)$, there exists $\left(\frac{y}{3}, 0\right) \in T(0, y)$

such that for all of these cases condition (2) holds for $k=\frac{3}{4}$.

From Theorem 1 it follows that $T$ has a fixed point in $X$, but $T$ is not a contraction mapping (for example, using the Chebyshev metric) on $X \subseteq \mathbb{R}^{2}$, thus we can not apply the classical Nadler's fixed point theorem.

Definition 4. Let $(X, d, E)$ be a vector metric space. The operator $T: X \rightarrow$ $P_{c l}(X)$ is said to be a multivalued $(a, b, c)$-contraction, if and only if $a, b, c \in \mathbb{R}_{+}$ with $a+b+c<1$ and for any $x, y \in X$ and any $u \in T(x)$, there exists $v \in T(y)$ such that

$$
d(u, v) \leq a d(x, y)+b d(x, u)+c d(y, v) .
$$

Theorem 2. Let $(X, d, E)$ be an E-complete vector metric space with $E$ Archimedean and let $T: X \rightarrow P_{c l}(X)$ be a multivalued $(a, b, c)$-contraction. Then $T$ has a fixed point in $X$ and for any $x \in X$, there exists a sequence of successive approximations of $T$ starting from $(x, y) \in \operatorname{Graph}(T)$ which $E$-converges in $(X, d, E)$ to the fixed point of $T$.

Proof. Let $x_{0} \in X$ such that $x_{1} \in T x_{0}$. Then there exists $x_{2} \in T x_{1}$ such that $d\left(x_{1}, x_{2}\right) \leq a d\left(x_{0}, x_{1}\right)+b d\left(x_{0}, x_{1}\right)+c d\left(x_{1}, x_{2}\right)$, where $a, b, c \in \mathbb{R}_{+}$with $a+$ $b+c<1$. Thus, define the sequence $\left(x_{n}\right) \in X$ by $x_{n+1} \in T x_{n}$ and $d\left(x_{n}, x_{n+1}\right) \leq$ $(a+b) d\left(x_{n-1}, x_{n}\right)+c d\left(x_{n}, x_{n+1}\right)$, for any $n \in \mathbb{N}^{*}$. Inductively, we obtain

$$
d\left(x_{n}, x_{n+1}\right) \leq \frac{a+b}{1-c} d\left(x_{n-1}, x_{n}\right) \leq \ldots \leq\left(\frac{a+b}{1-c}\right)^{n} d\left(x_{0}, x_{1}\right) \text {, for any } n \in \mathbb{N}^{*} \text {. }
$$

Since $a, b, c \in \mathbb{R}_{+}$with $a+b+c<1$, we have $0 \leq a+b<1-c$. We denote $k:=$ $\frac{a+b}{1-c} \in[0,1)$.

We have

$$
\begin{aligned}
d\left(x_{n}, x_{n+p}\right) & \leq d\left(x_{n}, x_{n+1}\right)+d\left(x_{n+1}, x_{n+2}\right)+\ldots+d\left(x_{n+p-1}, x_{n+p}\right) \\
& \leq\left(k^{n}+k^{n+1}+\ldots+k^{n+p-1}\right) d\left(x_{0}, x_{1}\right)=k^{n} \frac{1-k^{p}}{1-k} d\left(x_{0}, x_{1}\right) \\
& \leq \frac{k^{n}}{1-k} d\left(x_{0}, x_{1}\right)=a_{n} \cdot a:=b_{n}, \text { for any } n \in \mathbb{N}^{*} \text { and } p \in \mathbb{N}^{*},
\end{aligned}
$$


where $a_{n}=\frac{k^{n}}{1-k} \downarrow 0$ and $a=d\left(x_{0}, x_{1}\right) \in E^{+}$.

On the other hand, by $E$ Archimedean hypothesis, we have $b_{n} \downarrow 0$. So, the sequence $\left(x_{n}\right)$ is $E$-Cauchy în $X$. By the $E$-completness of $X$, there is $z \in X$ such that $x_{n} \stackrel{d, E}{\longrightarrow}$ $z$. Hence, there exists $\left(a_{n}\right)$ in $E$ such that $a_{n} \downarrow 0$ and $d\left(x_{n}, z\right) \leq a_{n}$.

We know $x_{n+1} \in T x_{n}$ for any $n \in \mathbb{N}$ and by the multivalued $(a, b, c)$-contraction condition it follows that there exists $u \in T z$ such that

$$
d\left(x_{n+1}, u\right) \leq a d\left(x_{n}, z\right)+b d\left(x_{n}, x_{n+1}\right)+c d(z, u) \text {, for any } n \in \mathbb{N} .
$$

Since

$$
\begin{aligned}
d(z, u) & \leq d\left(x_{n+1}, u\right)+d\left(x_{n+1}, z\right) \\
& \leq a d\left(x_{n}, z\right)+b d\left(x_{n}, x_{n+1}\right)+c d(z, u)+a_{n+1} \\
& \leq(a+1) a_{n}+b d\left(x_{n}, x_{n+1}\right)+c d(z, u), \text { hence } \\
d(z, u) & \leq \frac{a+1}{1-c} a_{n}+\frac{b}{1-c} d\left(x_{n}, x_{n+1}\right) \downarrow 0 .
\end{aligned}
$$

Thus, we have there exists $z=u \in T z$, i.e., $T$ has a fixed point in $X$.

Example 3. Let $E=\mathbb{R}^{2}$ and consider the set $X$, the mapping $d: X \times X \rightarrow E$ and the multivalued operator $T: X \rightarrow P_{c l}(X)$ be defined as in Example 2. It is easily to seen that $T$ is a multivalued $\left(\frac{1}{7}, \frac{1}{7}, \frac{2}{3}\right)$ contraction. From Theorem 2 it follows that that $T$ has a fixed point in $X$, but $T$ is not a $\left(\frac{1}{7}, \frac{1}{7}, \frac{2}{3}\right)$ contraction mapping with respect to the Chebyshev metric on $X \subseteq \mathbb{R}^{2}$. Thus we can not apply the classical Reich's fixed point theorem.

Remark 1. Theorem 1 and Theorem 2 generalize several known results in the theory of fixed points (see Nadler [17], Covitz-Nadler [14], Reich [15], Bucur, Guran, Petrussel [3]), etc. Notice also that here we do not need a closed graph condition on $T$, as in [3], for example.

\section{THE THEORY OF THE CONTRACTION PRINCIPLE IN VECTOR METRIC SPACES}

Our aim is to construct a theory of the fixed point theorems for multivalued operators in vector metric spaces. The novelity is given by a study of several properties of fixed points such as: the existence of fixed points and strict fixed points, data dependence of fixed points, convergence of the fixed point sets for a sequence of multivalued operators, Ulam stability of the inclusion $x \in T(x)$, well-posedness property of the fixed point problem, limit shadowing property of the multivalued operator and others, using the approach introduced in [16] and [14]. This theory can be similarly considered for fixed point theorems for classes of multivalued generalized contractions in vector metric spaces. 
Definition 5. Let $X, Y$ be two nonempty subsets and $T: X \rightarrow P(Y)$ be a multivalued operator. Then a singlevalued operator $t: X \rightarrow Y$ is called a selection of $T$ if and only if $t(x) \in T(x)$, for any $x \in X$.

Definition 6. Let $(X, d, E)$ be a vector metric space. Then, $T: X \rightarrow P(X)$ is called a multivalued weakly Picard operator (briefly $M W P$ operator) if for each $x \in X$ and each $y \in T(x)$ there exists a sequence $\left(x_{n}\right)_{n \in \mathbb{N}}$ in $X$ such that:

i) $x_{0}=x, x_{1}=y$;

ii) $x_{n+1} \in T\left(x_{n}\right)$, for all $n \in \mathbb{N}$;

i i i ) the sequence $\left(x_{n}\right)_{n \in \mathbb{N}}$ is $E$-convergent and its limit is a fixed point of $T$.

Definition 7. Let $(X, d, E)$ be a vector metric space and $T: X \rightarrow P(X)$ be a $M W P$ operator. Then we define the multivalued operator $T^{\infty}: \operatorname{Graph}(T) \rightarrow P\left(F_{T}\right)$ by the formula $\left\{T^{\infty}(x, y)=z \in F_{T}\right.$ : there exists a sequence of successive approximations of $T$ starting from $(x, y)$ that $E$-converges to $z$.

Definition 8. Let $(X, d, E)$ be a vector metric space and $T: X \rightarrow P(X)$ a $M W P$ operator. Then $T$ is said to be a $c$-multivalued weakly Picard operator (briefly $c$ $M W P$ operator) if and only if there exists a selection $t^{\infty}$ of $T^{\infty}$ such that $d\left(x, t^{\infty}(x, y)\right) \leq c d(x, y)$, for all $(x, y) \in \operatorname{Graph}(T)$.

Definition 9. Let $(X, d, E)$ be a vector metric space and let $\left(T_{n}\right)_{n \in \mathbb{N}}$ be a sequence of ordered sets in $P_{c l}(X)$. Then $T_{n}$ is called Hausdorff $E$-convergent to an ordered and closed set $T$ of $X$, denoted by $T_{n} \stackrel{H, d, E}{\longrightarrow} T$ as $n \rightarrow \infty$ if and only if there exists a sequence $\left(a_{n}\right) \subset E$ such that $a_{n} \downarrow 0$ as $n \rightarrow \infty$ and for any $u_{n} \in T_{n}(x)$, there exists $v \in T(x)$ (respectively for any $v \in T(x)$, there exists $\left.u_{n} \in T_{n}(x)\right)$ such that

$$
d\left(u_{n}, v\right) \leq a_{n}, \text { for any } n \in \mathbb{N} .
$$

Definition 10. Let $(X, d, E)$ be an $E$-complete vector metric space and $T: X \rightarrow$ $P(X)$. By definition, $T$ is called a multivalued Picard operator (briefly $M P$ operator) if and only if:

i) $(S F)_{T}=F_{T}=\left\{x^{*}\right\}$;

ii) $T^{n}(x) \stackrel{H, d, E}{\longrightarrow}\left\{x^{*}\right\}$ as $n \rightarrow \infty$, for each $x \in X$.

Lemma 1 (Extended Cauchy Lemma). Let $E$ be an order complete Riesz space. Let $a_{n} \in \mathbb{R}_{+}, b_{n} \in E^{+}, n \in \mathbb{N}^{*}$ such that $\sum_{i=0}^{\infty}\left|a_{i}\right|<+\infty$ and $b_{n} \stackrel{o}{\longrightarrow} 0$ as $n \rightarrow \infty$. Then

$$
\sum_{i=0}^{n} a_{n-i} b_{i} \stackrel{o}{\longrightarrow} 0 \text { as } n \rightarrow \infty
$$


Proof. We split the above sum in two parts:

$$
\sum_{i=0}^{n} a_{n-i} b_{i}=\sum_{i=0}^{\left[\frac{n}{2}\right]} a_{n-i} b_{i}+\sum_{i=\left[\frac{n}{2}\right]+1}^{n} a_{n-i} b_{i}
$$

From $b_{i} \stackrel{o}{\longrightarrow} 0$ as $i \rightarrow \infty$ it follows that there exists a sequence $c_{i} \downarrow 0$ in $E$ such that $\left|b_{i}\right| \leq c_{i}$ holds for all $i$.

For the first part of the sum we have:

$$
\begin{aligned}
\left|\sum_{i=0}^{\left[\frac{n}{2}\right]} a_{n-i} b_{i}\right| & \leq \sum_{i=0}^{\left[\frac{n}{2}\right]}\left|a_{n-i}\right|\left|b_{i}\right| \leq \sum_{i=0}^{\left[\frac{n}{2}\right]}\left|a_{n-i}\right| c_{i} \\
& =\sum_{i=0}^{\left[\frac{n}{2}\right]}\left|a_{n-i}\right| c_{0} \leq c_{0} \sum_{i=\left[\frac{n}{2}\right]+1}^{\infty}\left|a_{i}\right|:=x_{n} \downarrow 0 \text { as } n \rightarrow \infty,
\end{aligned}
$$

since $E$ is Archimedean.

Since $c_{i} \downarrow 0$ as $i \rightarrow \infty$, we have there exists $m_{n}:=\sup \left\{c_{i}: i \geq\left[\frac{n}{2}\right]+1\right\} \downarrow 0$ as $n \rightarrow \infty$, because the space $E$ is order complete.

For the second part of the sum we have:

$$
\begin{aligned}
\left|\sum_{i=\left[\frac{n}{2}\right]+1}^{n} a_{n-i} b_{i}\right| & \leq \sum_{i=\left[\frac{n}{2}\right]+1}^{n}\left|a_{n-i}\right|\left|b_{i}\right| \\
& \leq \sum_{i=\left[\frac{n}{2}\right]+1}^{n}\left|a_{n-i}\right| c_{i} \leq m_{n} \sum_{i=0}^{\infty}\left|a_{i}\right|:=y_{n} \downarrow 0 \text { as } n \rightarrow \infty .
\end{aligned}
$$

Since

$$
\left|\sum_{i=0}^{n} a_{n-i} b_{i}\right| \leq\left|\sum_{i=0}^{\left[\frac{n}{2}\right]} a_{n-i} b_{i}\right|+\left|\sum_{i=\left[\frac{n}{2}\right]+1}^{n} a_{n-i} b_{i}\right| \leq x_{n}+y_{n}=z_{n} \downarrow 0 \text { as } n \rightarrow \infty,
$$

thus

$$
\sum_{i=0}^{n} a_{n-i} b_{i} \stackrel{o}{\longrightarrow} 0 \text { as } n \rightarrow \infty
$$


Theorem 3. Let $(X, d, E)$ be an $E$-complete vector metric space with $E$ Archimedean and let $T: X \rightarrow P_{c l}(X)$ a multivalued $k$-contraction. Then the following statements hold:

i) $F_{T} \neq \varnothing$;

ii) $T$ is a $\frac{1}{1-k}$-multivalued weakly Picard operator;

ii i) Let $S: X \rightarrow P_{c l}(X)$ be a multivalued $k$-contraction and $\eta \in E^{+}$such that for any $u \in S(x)$, there exists $v \in T(x)$ such that $d(u, v) \leq \eta$ (respectively for any $u \in T(x)$, there exists $v \in S(x)$ such that $d(u, v) \leq \eta)$. Then for any $p \in F_{S}$, there exists $q \in F_{T}$ such that $d(p, q) \leq \frac{1}{1-k} \eta$ (respectively for any $p \in F_{T}$, there exists $q \in F_{S}$ such that $\left.d(p, q) \leq \frac{1}{1-k} \eta\right)$.

iv) Let $T_{n}: X \rightarrow P_{c l}(X), n \in \mathbb{N}$ be a sequence of multivalued $k$-contractions such that $T_{n}(x) \stackrel{H, d, E}{\longrightarrow} T(x)$ as $n \rightarrow \infty$, uniformly with respect to $x \in X$. Then, $F_{T_{n}} \stackrel{H, d, E}{\longrightarrow} F_{T}$ as $n \rightarrow \infty$.

v) (Ulam-Hyers stability of the inclusion $x \in T(x))$ Let $\epsilon \in E^{+}$be such that there exists $y \in T(x): d(x, y) \leq \epsilon$. Then, there exists $x^{*} \in F_{T}$ such that $d\left(x, x^{*}\right) \leq \frac{1}{1-k} \epsilon$.

Proof. $i$ ) It follows from Theorem 1.

ii) Let $x_{0}=x, x_{1}=y \in X$ and define the sequence $x_{n+1} \in T\left(x_{n}\right)$, for all $n \in \mathbb{N}$, which from Theorem $1 E$-converges in $(X, d, E)$ to $x^{*}$, for all $x \in X$ and $F_{T} \neq \varnothing$. We know that $d\left(x_{n}, x_{n+p}\right) \leq k^{n} \frac{1-k^{p}}{1-k} d\left(x_{0}, x_{1}\right)$, for any $n \in \mathbb{N}^{*}$ and $p \in \mathbb{N}^{*}$ and letting $p \rightarrow \infty$, we get that

$$
d\left(x_{n}, x^{*}\right) \leq \frac{k^{n}}{1-k} d\left(x_{0}, x_{1}\right) \text {, for any } n \in \mathbb{N}^{*} .
$$

For $n=1$, we get

$$
d\left(x_{1}, x^{*}\right) \leq \frac{k}{1-k} d\left(x_{0}, x_{1}\right) .
$$

Then

$$
d\left(x_{0}, x^{*}\right) \leq d\left(x_{0}, x_{1}\right)+d\left(x_{1}, x^{*}\right) \leq \frac{1}{1-k} d\left(x_{0}, x_{1}\right) .
$$

Hence, $T$ is a $\frac{1}{1-k}$-multivalued weakly Picard operator.

iii) Let $x_{0} \in F_{S}$ be arbitrary chosen. Then, by $i i$ ), we have that there exists $t^{\infty}\left(x_{0}, x_{1}\right) \in F_{T}$ such that

$$
d\left(x_{0}, t^{\infty}\left(x_{0}, x_{1}\right)\right) \leq \frac{1}{1-k} d\left(x_{0}, x_{1}\right), \text { for any } x_{1} \in T\left(x_{0}\right) .
$$

Since there exists $x_{1} \in T\left(x_{0}\right)$ such that $d\left(x_{0}, x_{1}\right) \leq \eta$, we have that

$$
d\left(x_{0}, t^{\infty}\left(x_{0}, x_{1}\right)\right) \leq \frac{1}{1-k} \eta .
$$


By a similar procedure we can prove that, for any $y_{0} \in F_{T}$ there exists $s^{\infty}\left(y_{0}, y_{1}\right) \in$ $F_{S}$ such that

$$
d\left(y_{0}, s^{\infty}\left(y_{0}, y_{1}\right)\right) \leq \frac{1}{1-k} \eta .
$$

$i v)$ Since $T_{n}(x) \stackrel{H, d, E}{\longrightarrow} T(x)$ as $n \rightarrow \infty$, thus there exists $\left(\eta_{n}\right)$ in $E$ such that $\eta_{n} \downarrow 0$ and for any $u_{n} \in T_{n}(x)$, there exists $v \in T(x)$ (respectively for any $v \in T(x)$, there exists $\left.u_{n} \in T_{n}(x)\right)$ such that

$$
d\left(u_{n}, v\right) \leq \eta_{n}, \text { for any } n \in \mathbb{N} .
$$

By $i$ i $i$ ), follows immediately that for any $x_{0_{n}} \in F_{T_{n}}$ (respectively for any $y_{0} \in F_{T}$ ) we have that there exists $t^{\infty}\left(x_{0_{n}}, x_{1}\right) \in F_{T}$ (respectively, there exists $s^{\infty}\left(y_{0}, y_{1_{n}}\right) \in$ $F_{T_{n}}$ ) such that

$$
\begin{aligned}
& d\left(x_{0_{n}}, t^{\infty}\left(x_{0_{n}}, x_{1}\right)\right) \leq \frac{1}{1-k} \eta_{n}, \text { for any } n \in \mathbb{N}, \text { respectively } \\
& d\left(y_{0}, s^{\infty}\left(y_{0}, y_{1_{n}}\right)\right) \leq \frac{1}{1-k} \eta_{n}, \text { for any } n \in \mathbb{N} .
\end{aligned}
$$

Letting in (3.1) $n \rightarrow \infty$, we obtain that there exists $a_{n}:=\frac{1}{1-k} \eta_{n} \subset E$ such that $a_{n} \downarrow 0$ as $n \rightarrow \infty$ and for any $p_{n}:=x_{0_{n}} \in F_{T_{n}}$, there exists $q:=t^{\infty}\left(x_{0_{n}}, x_{1}\right) \in F_{T}$ (respectively for any $q:=y_{0} \in F_{T}$, there exists $\left.p_{n}:=s^{\infty}\left(y_{0}, y_{1_{n}}\right) \in F_{T_{n}}\right)$ such that

$$
d\left(p_{n}, q\right) \leq a_{n} \text {, for any } n \in \mathbb{N} \text {. }
$$

Hence, $F_{T_{n}} \stackrel{H, d, E}{\longrightarrow} F_{T}$ as $n \rightarrow \infty$.

v) Let $\epsilon \in E^{+}$and $x \in X$. By hypothesis, there exists $y \in T(x)$ such that $d(x, y) \leq \epsilon$. By the proof of $i i)$, we have that there exists $x^{*}:=t^{\infty}(x, y) \in F_{T}$ such that

$$
d\left(x, x^{*}\right) \leq \frac{1}{1-k} d(x, y) \leq \frac{1}{1-k} \epsilon .
$$

A second result for multivalued contractions in vector metric spaces is as follows.

Theorem 4. Let $(X, d, E)$ be an E-complete vector metric space with $E$ Archimedean and let $T: X \rightarrow P_{c l}(X)$ a multivalued $k$-contraction with $(S F)_{T} \neq \varnothing$. Then the following statements hold:

i) $F_{T}=(S F)_{T}=\left\{x^{*}\right\}$;

ii) $F_{T^{n}}=(S F)_{T^{n}}=\left\{x^{*}\right\}$ for $n \in \mathbb{N}^{*}$;

iii) $T^{n}(x) \stackrel{H, d, E}{\longrightarrow}\left\{x^{*}\right\}$ as $n \rightarrow \infty$, for each $x \in X$;

iv) Let $S: X \rightarrow P_{c l}(X)$ a multivalued operator and $\eta \in E^{+}$such that $F_{S} \neq \varnothing$ and for any $u \in S(x)$, there exists $v \in T(x)$ such that $d(u, v) \leq \eta$ (respectively for any $u \in T(x)$, there exists $v \in S(x)$ such that $d(u, v) \leq \eta)$. Then for any $p \in F_{S}$, 
there exists $q \in F_{T}$ such that $d(p, q) \leq \frac{1}{1-k} \eta$ (respectively for any $p \in F_{T}$, there exists $q \in F_{S}$ such that $\left.d(p, q) \leq \frac{1}{1-k} \eta\right)$;

v) Let $T_{n}: X \rightarrow P_{c l}(X), n \in \mathbb{N}$ be a sequence of multivalued operators such that $F_{T_{n}} \neq \varnothing$ for each $n \in \mathbb{N}$ and $T_{n}(x) \stackrel{H, d, E}{\longrightarrow} T(x)$ as $n \rightarrow \infty$, uniformly with respect to $x \in X$. Then, $F_{T_{n}} \stackrel{H, d, E}{\longrightarrow} F_{T}$ as $n \rightarrow \infty$.

vi) (Well-posedness property of the fixed point problem) If $\left(x_{n}\right)_{n \in \mathbb{N}}$ is a sequence in $X$ such that there exists $y_{n} \in T\left(x_{n}\right), n \in \mathbb{N}$ with the property

$$
d\left(x_{n}, y_{n}\right) \stackrel{o}{\longrightarrow} 0 \text {, as } n \rightarrow \infty,
$$

then $x_{n} \stackrel{d, E}{\longrightarrow} x^{*}$, as $n \rightarrow \infty$.

vii) (Limit shadowing property of the multivalued operator) If $\left(y_{n}\right)_{n \in \mathbb{N}}$ is a sequence in $X$ such that there exists $u_{n} \in T\left(y_{n}\right), n \in \mathbb{N}$ with the property

$$
d\left(y_{n+1}, u_{n}\right) \stackrel{o}{\longrightarrow} 0 \text {, as } n \rightarrow \infty,
$$

then there exists a sequence $\left(x_{n}\right)_{n \in \mathbb{N}} \subset X$ of successive approximations for $T$, such that

$$
d\left(x_{n}, y_{n}\right) \stackrel{o}{\longrightarrow} 0 \text {, as } n \rightarrow \infty .
$$

Proof. $i$ ) Let $x^{*} \in(S F)_{T}$ and we prove that $x^{*}$ is the unique strict fixed point of $T$.

We suppose there exists $y \in(S F)_{T}, y \neq x^{*}$, then $T(y)=\{y\}$.

From

$$
d\left(x^{*}, y\right)=d\left(T\left(x^{*}\right), T(y)\right) \leq k d\left(x^{*}, y\right),
$$

we obtain the contradiction $k \geq 1$. Thus, $(S F)_{T}=\left\{x^{*}\right\}$.

We know that $\left\{x^{*}\right\} \subset F_{T}$ and prove that $F_{T} \subset\left\{x^{*}\right\}$.

Let $y \in F_{T}, y \neq x^{*}$. Since $x^{*}, y \in X$ and for any $u:=y \in T(y)$, there exists $v \in T\left(x^{*}\right)=\left\{x^{*}\right\}$ such that

$$
d\left(y, x^{*}\right) \leq k d\left(x^{*}, y\right),
$$

we obtain the contradiction $k \geq 1$. Thus, $F_{T} \subset\left\{x^{*}\right\}$.

Hence, $F_{T}=\left\{x^{*}\right\}$.

ii) Let $x^{*} \in(S F)_{T^{n}}$, for $n \in \mathbb{N}^{*}$ and we prove that $x^{*}$ is the unique strict fixed point of $T^{n}$.

We suppose there exists $y \in(S F)_{T^{n}}, y \neq x^{*}$, then $T^{n}(y)=\{y\}$, for $n \in \mathbb{N}^{*}$.

Let $x^{*}, y \in X$ and for any $u_{1} \in T(y)$, there exists $v_{1} \in T\left(x^{*}\right)$ such that

$$
d\left(u_{1}, v_{1}\right) \leq k d\left(x^{*}, y\right) .
$$

Let $u_{1}, v_{1} \in X$ and for any $u_{2} \in T\left(u_{1}\right) \subset T(T(y))=T^{2}(y)$, there exists $v_{2} \in$ $T\left(v_{1}\right) \subset T\left(T\left(x^{*}\right)\right)=T^{2}\left(x^{*}\right)$ such that

$$
d\left(u_{2}, v_{2}\right) \leq k d\left(u_{1}, v_{1}\right) \leq k^{2} d\left(x^{*}, y\right) .
$$


Inductively, let $u_{n-1}, v_{n-1} \in X, n \geq 2$ and for any $u_{n} \in T\left(u_{n-1}\right) \subset T^{n}(y)=\{y\}$, there exists $v_{n} \in T\left(v_{n-1}\right) \subset T^{n}\left(x^{*}\right)=\left\{x^{*}\right\}$ such that

$$
d\left(y, x^{*}\right) \leq k^{n} d\left(x^{*}, y\right) \text {, for any } n \in \mathbb{N}^{*} .
$$

Thus, $y=x^{*}$ and $(S F)_{T^{n}}=\left\{x^{*}\right\}$.

We know that $\left\{x^{*}\right\} \subset F_{T^{n}}$ and prove that $F_{T^{n}} \subset\left\{x^{*}\right\}$.

Let $y \in F_{T^{n}}, y \neq x^{*}$. Similarly, we have that for any $x^{*}, y \in X$ and for any $u_{n}:=$ $y \in T^{n}(y)$, there exists $v_{n} \in T^{n}\left(x^{*}\right)=\left\{x^{*}\right\}$ such that

$$
d\left(y, x^{*}\right) \leq k^{n} d\left(x^{*}, y\right) \text {, for any } n \in \mathbb{N}^{*} .
$$

Thus, $y=x^{*}$ and hence $F_{T^{n}}=(S F)_{T^{n}}=\left\{x^{*}\right\}$.

ii i i) Let $x, x^{*} \in X$ and for any $u_{1}:=x \in T(x)$, there exists $v_{1} \in T\left(x^{*}\right)$ such that

$$
d\left(u_{1}, v_{1}\right) \leq k d\left(x, x^{*}\right) .
$$

Let $u_{1}, v_{1} \in X$ and for any $u_{2} \in T\left(u_{1}\right) \subset T(T(x))=T^{2}(x)$, there exists $v_{2} \in$ $T\left(v_{1}\right) \subset T\left(T\left(x^{*}\right)\right)=T^{2}\left(x^{*}\right)$ such that

$$
d\left(u_{2}, v_{2}\right) \leq k d\left(u_{1}, v_{1}\right) \leq k^{2} d\left(x, x^{*}\right) .
$$

Inductively, let $u_{n-1}, v_{n-1} \in X, n \geq 2$ and for any $u_{n} \in T\left(u_{n-1}\right) \subset T^{n}(x)$, there exists $v_{n} \in T\left(v_{n-1}\right) \subset T^{n}\left(x^{*}\right)=\left\{x^{*}\right\}$ such that

$$
d\left(u_{n}, v_{n}\right) \leq k^{n} d\left(x, x^{*}\right) \text {, for any } n \in \mathbb{N}^{*} .
$$

By a similar procedure, we can obtain that for any $u_{n-1}, v_{n-1} \in X, n \geq 2$ and for any $u_{n} \in T\left(v_{n-1}\right) \subset T^{n}\left(x^{*}\right)=\left\{x^{*}\right\}$, there exists $v_{n} \in T\left(v_{u-1}\right) \subset T^{n}(x)$ such that

$$
d\left(u_{n}, v_{n}\right) \leq k^{n} d\left(x^{*}, x\right) \text {, for any } n \in \mathbb{N}^{*} .
$$

Letting in (3.2) and (3.3) $n \rightarrow \infty$, we obtain that there exists $a_{n}:=k^{n} d\left(x^{*}, x\right) \subset E$ such that $a_{n} \downarrow 0$ as $n \rightarrow \infty$ and hence $T^{n}(x) \stackrel{H, d, E}{\longrightarrow}\left\{x^{*}\right\}$ as $n \rightarrow \infty$, for each $x \in X$.

$i v)$ Let $y \in F_{S}$. Then, there exists $x^{*} \in T(y)$ such that

$$
d\left(y, x^{*}\right) \leq d(y, x)+d\left(x, x^{*}\right) \text {, for any } x \in T(y) .
$$

Since, for any $x \in T(y)$, there exists $x^{*} \in T\left(x^{*}\right)=\left\{x^{*}\right\}$ such that $d\left(x, x^{*}\right) \leq$ $k d\left(y, x^{*}\right)$, we obtain

$$
d\left(y, x^{*}\right) \leq \eta+k d\left(y, x^{*}\right)
$$

and thus,

$$
d\left(y, x^{*}\right) \leq \frac{1}{1-k} \eta .
$$

Hence, for any $y \in F_{S}$, there exists $x^{*} \in F_{T}$ such that

$$
d\left(y, x^{*}\right) \leq \frac{1}{1-k} \eta \text {. }
$$


By a similar procedure, we obtain that for any $y \in F_{T}$, there exists $x^{*} \in F_{S}$ such that

$$
d\left(y, x^{*}\right) \leq \frac{1}{1-k} \eta \text {. }
$$

$v$ ) It follows from $i v$ ) (see also, Theorem $3 i v$ )).

vi) Let $x_{n}, x^{*} \in X, n \in \mathbb{N}$. Then, for any $y_{n} \in T\left(x_{n}\right)$, there exists $u \in T\left(x^{*}\right)=$ $\left\{x^{*}\right\}$ such that

Since

$$
d\left(y_{n}, x^{*}\right) \leq k d\left(x_{n}, x^{*}\right) .
$$

$$
d\left(x_{n}, x^{*}\right) \leq d\left(x_{n}, y_{n}\right)+d\left(y_{n}, x^{*}\right) \leq d\left(x_{n}, y_{n}\right)+k d\left(x_{n}, x^{*}\right),
$$

thus

$$
d\left(x_{n}, x^{*}\right) \leq \frac{1}{1-k} d\left(x_{n}, y_{n}\right), \text { for any } n \in \mathbb{N} .
$$

By $d\left(x_{n}, y_{n}\right) \stackrel{o}{\longrightarrow} 0$ as $n \rightarrow \infty$, we obtain that there exists $\left(a_{n}\right)$ in $E$ such that $a_{n} \downarrow 0$ as $n \rightarrow \infty$ and

$$
\left|d\left(x_{n}, y_{n}\right)\right| \leq a_{n} \text {, for any } n \in \mathbb{N} .
$$

Letting in (3.4) $n \rightarrow \infty$ and using (3.5), we have

$$
d\left(x_{n}, x^{*}\right) \leq \frac{1}{1-k} a_{n} \downarrow 0, \text { as } n \rightarrow \infty .
$$

Hence, $x_{n} \stackrel{d, E}{\longrightarrow} x^{*}$ as $n \rightarrow \infty$.

vi i) Let $y_{n}, x^{*} \in X, n \in \mathbb{N}$. Then, for any $u_{n} \in T\left(y_{n}\right)$, there exists $v_{n} \in T\left(x^{*}\right)=$ $\left\{x^{*}\right\}$ such that

Then:

$$
d\left(u_{n}, x^{*}\right) \leq k d\left(y_{n}, x^{*}\right) .
$$

$$
\begin{aligned}
d\left(y_{n+1}, x^{*}\right) & \leq d\left(y_{n+1}, u_{n}\right)+d\left(u_{n}, x^{*}\right) \leq d\left(y_{n+1}, u_{n}\right)+k d\left(y_{n}, x^{*}\right) \\
& \leq d\left(y_{n+1}, u_{n}\right)+k\left[d\left(y_{n}, u_{n-1}\right)+k d\left(y_{n-1}, x^{*}\right)\right] \\
& =d\left(y_{n+1}, u_{n}\right)+k d\left(y_{n}, u_{n-1}\right)+k^{2} d\left(y_{n-1}, x^{*}\right)
\end{aligned}
$$

and thus, inductively we get that:

$$
\begin{aligned}
d\left(y_{n+1}, x^{*}\right) & \leq d\left(y_{n+1}, u_{n}\right)+k d\left(y_{n}, u_{n-1}\right)+\ldots+k^{n} d\left(y_{1}, u_{0}\right)+k^{n+1} d\left(y_{0}, x^{*}\right) \\
& =\sum_{i=0}^{n} k^{n-i} d\left(y_{i+1}, u_{i}\right)+k^{n+1} d\left(y_{0}, x^{*}\right) \stackrel{o}{\longrightarrow} 0 \text { as } n \rightarrow \infty,
\end{aligned}
$$

which follows from Lemma 1.

By $o$-convergence definition to 0 , we have there exists $\left(a_{n}\right)$ in $E$ such that $a_{n} \downarrow 0$ as $n \rightarrow \infty$ and $\left|d\left(y_{n+1}, x^{*}\right)\right| \leq a_{n+1}$, for any $n \in \mathbb{N}$.

On the other hand, by the proof of Theorem $3 i$ )-ii), we know that there exists a sequence $\left(x_{n}\right)_{n \in \mathbb{N}}$ of successive approximations for $T$ starting from arbitrary $(x, y) \in$ $\operatorname{Graph}(T)$ which $E$-converge to a fixed point $x^{*} \in X$ of the operator $T$. Since, the 
fixed point is unique, we get that $d\left(x_{n}, x^{*}\right) \stackrel{o}{\longrightarrow} 0$ as $n \rightarrow \infty$ (by definition, there exists $\left(b_{n}\right)$ in $E$ such that $b_{n} \downarrow 0$ as $n \rightarrow \infty$ and $\left|d\left(x_{n}, x^{*}\right)\right| \leq b_{n}$, for any $\left.n \in \mathbb{N}\right)$. Thus, for such a sequence $\left(x_{n}\right)_{n \in \mathbb{N}}$, we have

$\left|d\left(x_{n}, y_{n}\right)\right| \leq\left|d\left(x_{n}, x^{*}\right)\right|+\left|d\left(x^{*}, y_{n}\right)\right| \leq\left(b_{n}+a_{n}\right) \downarrow 0$ as $n \rightarrow \infty$, for each $x \in X$.

Hence,

$$
d\left(x_{n}, y_{n}\right) \stackrel{o}{\longrightarrow} 0, \text { as } n \rightarrow \infty .
$$

A third result for multivalued $k$-contractions in vector metric spaces is the following.

Theorem 5. Let $(X, d, E)$ be an E-complete vector metric space with $E$ Archimedean and let $T: X \rightarrow P_{c p}(X)$ be a multivalued $k$-contraction such that $T\left(F_{T}\right)=F_{T}$. Then the following statements hold:

i) $T(x)=F_{T}$, for each $x \in F_{T}$;

ii) If $\left(x_{n}\right)_{n \in \mathbb{N}} \subset X$ is a sequence such that $x_{n} \stackrel{d, E}{\longrightarrow} x^{*} \in F_{T}$ as $n \rightarrow \infty$, then $T\left(x_{n}\right) \stackrel{H, d, E}{\longrightarrow} F_{T}$ as $n \rightarrow \infty$.

Proof. $i)$ Let $x \in F_{T}$ be arbitrary chosen. Then, $x \in T(x)$ and thus $F_{T} \subset T(x)$. On the other hand $T(x) \subset T\left(F_{T}\right) \subset F_{T}$.

Thus, $T(x)=F_{T}$, for each $x \in F_{T}$.

ii) Since $x_{n} \stackrel{d, E}{\longrightarrow} x^{*} \in F_{T}$ as $n \rightarrow \infty$, it follows that there exists a sequence $\left(a_{n}\right) \subset$ $E$ such that $a_{n} \downarrow 0$ as $n \rightarrow \infty$ and

$$
d\left(x_{n}, x^{*}\right) \leq a_{n}, \text { for any } n \in \mathbb{N} .
$$

Let $x_{n} \in X, n \in \mathbb{N}, x^{*} \in X$. Then, for any $u_{n} \in T\left(x_{n}\right)$, there exists $u \in T\left(x^{*}\right)=F_{T}$ (respectively, for any $u \in T\left(x^{*}\right)=F_{T}$, there exists $u_{n} \in T\left(x_{n}\right)$ ) such that

$$
d\left(u_{n}, u\right) \leq k d\left(x_{n}, x^{*}\right) \leq k a_{n} \downarrow 0, \text { as } n \rightarrow \infty,
$$

since $E$ is Archimedean. Hence, $T\left(x_{n}\right) \stackrel{H, d, E}{\longrightarrow} F_{T}$ as $n \rightarrow \infty$.

Open questions.

The above considerations give rise to the following tasks:

(a) To construct a similar theory for some multivalued generalized contraction in vector metric spaces.

(b) To give fixed point theorems for nonlinear contractions in vector metric spaces (see $[2,5,6,10,18,19])$.

\section{ACKNOWLEDGEMENT}

The author wishes to thank the referee for the valuable suggestions leading to an improved version of the manuscript. 


\section{REFERENCES}

[1] C. D. Aliprantis and K. C. Border, Infinite Dimensional Analysis. Berlin: Springer-Verlag, 1999.

[2] M. Berinde and V. Berinde, "On a class of multi-valued weakly Picard mappings," J. Math. Anal. Appl., vol. 326, pp. 772-782, 2007.

[3] A. Bucur, L. Guran, and A. Petruşel, "Fixed points for multivalued operators on a set endowed with vector-valued metrics and applications," Fixed Point Theory, vol. 10, no. 1, pp. 19-34, 2009.

[4] C. Çevik and I. Altun, "Vector metric spaces and some properties," Topol. Methods Nonlinear Anal., vol. 34, pp. 375-382, 2009.

[5] P. Z. Daffer and H. Kaneko, "Fixed points of generalized contractive multi-valued mappings," $J$. Math. Anal. Appl., vol. 192, pp. 655-666, 1995.

[6] A. Granas and J. Dugundji, Fixed Point Theory. New York: Springer-Verlag, 2003.

[7] I.-G. Huang and X. Zhang, "Cone metric spaces and fixed point theorems of contractive mappings," J. Math. Anal. Appl., vol. 332, pp. 1468-1476, 2007.

[8] H. Kaneko, "A general principle for fixed points of contractive multi-valued mappings," Math. Japon., vol. 31, pp. 407-411, 1986.

[9] H. Kaneko, "Generalized contractive multi-valued mappings and their fixed points," Math. Japon., vol. 33, pp. 57-64, 1988.

[10] N. Mizoguchi and W. Takahashi, "Fixed point theorems for multi-valued mappings on complete metric spaces," J. Math. Anal. Appl., vol. 141, pp. 177-188, 1989.

[11] I.-R. Petre, "Fixed points for $\varphi$-contractions in E-Banach spaces," Fixed Point Theory, vol. 13, no. 2, pp. 623-640, 2012.

[12] I.-R. Petre, "A multivalued version of krasnoselskii's theorem in generalized Banach spaces," An. St. Univ. Ovidius Constanta, vol. 22, no. 2, pp. 177-192, DOI: 10.2478/auom-2014-0041, 2014.

[13] I.-R. Petre and M. Bota, "Fixed point theorems on generalized b-metric spaces," Publ. Math. Debrecen, vol. 83, no. 1-2, pp. 139-159, DOI: 10.5486/PMD.2013.5554, 2013.

[14] A. Petrussel and I. A. Rus, "The theory of a metric fixed point theorem for multivalued operators," Fixed Point Theory and its Applications, pp. 167-176, 2010.

[15] S. Reich, "Fixed points of contractive functions," Boll. Un. Mat. Ital., vol. 5, no. 4, pp. 26-42, 1972.

[16] I. A. Rus, "The theory of a metrical fixed point theorem: Theoretical and applicative relevances," Fixed Point Theory, vol. 9, no. 2, pp. 541-559, 2008.

[17] I. A. Rus, A. Petruşel, and G. Petruşel, Fixed Point Theory. Cluj-Napoca: Cluj University Press, 2008.

[18] P. P. Zabrejko, "K-metric and K-normed linear spaces: survey," Collect. Math., vol. 48, no. 4-6, pp. 825-859, 1997.

[19] E. Zeidler, Nonlinear Functional Analysis. Berlin: Springer-Verlag, 1993, vol. I.

Author's address

Ioan-Radu Petre

Babeş-Bolyai University

Department of Applied Mathematics

Kogălniceanu Street, No. 1, 400084, Cluj-Napoca, Romania.

E-mail address: ioan. petre@ubbcluj.ro 